\title{
Mathematical model and influence factor analysis for productivity prediction of fractured-vuggy carbonate reservoir when drilling into cavern reservoir
}

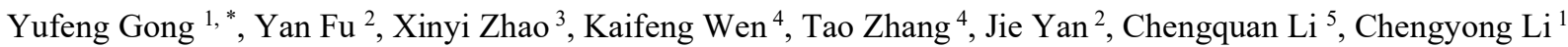 \\ ${ }^{1}$ School of Energy, Chengdu University of Technology, Chengdu, China \\ ${ }^{2}$ Engineering Technology Research Institute, PetroChina Southwest Oil \& Gas Field Company, Chengdu, China \\ ${ }^{3}$ China National Petroleum Corporation Southwest Oil and Gas Field Branch Chuanzhong Oil and Gas Mine, Suining, China \\ ${ }^{4}$ No. 2 Gas Production Plant, PetroChina ChangQing Oilfield Company, Yulin, China \\ ${ }^{5}$ Development Division, Southwest Oil and Gas Field Branch, China National Petroleum Corporation, Chengdu, China
}

\begin{abstract}
The seam hole type carbonate didn't touch type drill in the cave reservoir oil and gas resources is rich, but due to the large difference of unused reservoir capacity, capacity of main control factors and the evaluation model is not clear, so the reservoir is difficult to reasonable and effective development, in order to improve the cave reservoir development effect, find the factors influencing the cave reservoir capacity of the master, This article through to carry out the cave type carbonate reservoir capacity model test, the cave reservoir productivity prediction model is set up and analyze the factors affecting capacity, studies have shown that the wellbore storage coefficient and skin factor of cave type carbonate reservoir had a greater influence on the vertical well productivity, this paper research content for the cave type of carbonate reservoir reserves producing has a certain guiding significance.
\end{abstract}

\section{Preface}

Carbonate reservoirs are widely distributed in China, with $29.3 \times 10^{8} \mathrm{t}$ of proven petroleum reserves, which are indispensable for oil and gas exploration and development. Buried hill fracture-vuggy-type reservoirs are rich in untapped reserves. However, fracture-vuggy-type carbonate reservoirs are complex and diverse in their reservoir space. Fracture-vuggy carbonate reservoirs cannot be developed reasonably and effectively because of the strong heterogeneity of assemblage relationship and seepage field distribution and large difference in reservoir productivity.

In order to realize stable and high production and effective development of fracture-vuggy carbonate reservoir, this paper, based on the basic principle of seepage mechanics, establishes the productivity prediction model of fracturevuggy carbonate reservoir and analyzes the influencing factors of productivity. The research shows that the effects of wellbore reservoir coefficient, skin coefficient, reserve-volume ratio and interflow coefficient on single well productivity of vertical well in fracture-vuggy carbonate reservoir are analyzed, and the reliability of mathematical model established in this paper is verified by field data. The research results of this paper can provide support for reasonable and effective reserve production evaluation technology, and it is of great significance for stable and high production and enhanced oil recovery of fracture-vuggy carbonate reservoirs.

\section{Mathematical model of cavernous seepage through drilling}

\subsection{Establishment of physical model}

Suppose a circular reservoir, outer boundary distance $r_{e}(\mathrm{~m})$, formation thickness $h(\mathrm{~m})$; The reservoir presents a compound distribution of two zones. Zone 1 is a karst cave directly connected to the wellbore, and Zone 2 is a fracture-vuggy zone. The radius of hyperosmotic zone is $r_{1}(m)$. The low permeability zone is $k_{2}\left(10^{-3} \mu m^{2}\right)$, and the porosity is $\phi_{2}$. The formation fluid is slightly compressible, and the flow through the formation satisfies Darcy's law. The viscosity of the fluid is $\mu(\mathrm{mPa} \mathrm{s})$, and the comprehensive compressibility coefficients of zones 1 and 2 are $C_{t 1}$ and $C_{t 2}\left(\mathrm{MPa}^{-1}\right)$.The pressure of each reservoir and the cave is the original formation pressure $p_{i}(\mathrm{MPa})$. The wellbore radius is $r_{w}(\mathrm{~m})$, the production rate is $q\left(\mathrm{~m}^{3} / \mathrm{d}\right)$, and considering the well storage and skin effects, the wellbore storage coefficient is $\mathrm{C}\left(\mathrm{m}^{3} / \mathrm{MPa}\right)$, and the skin coefficient is $\mathrm{S}$. The influence of gravity and capillary force is not considered.

\footnotetext{
* Corresponding author: gongyufeng1995@stu.cdut.edu.cn
} 


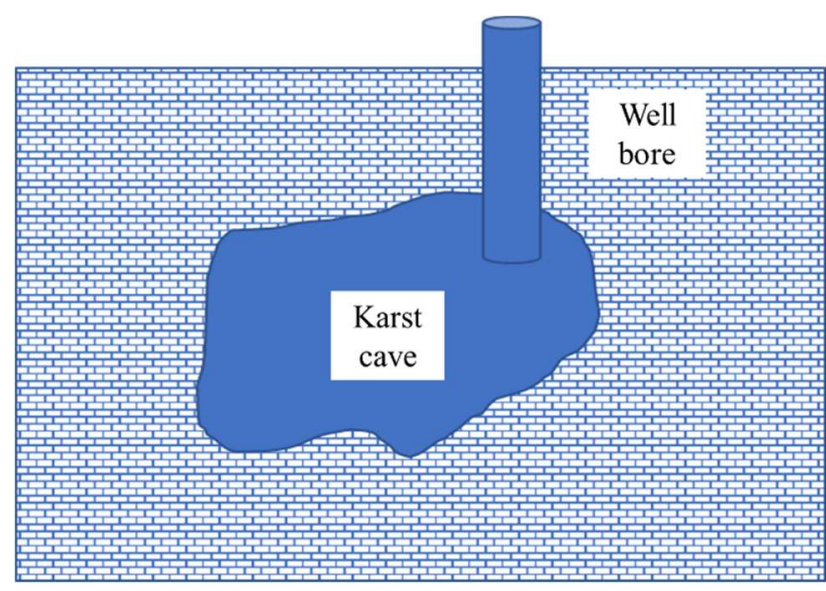

Figure 1. Physical model of cavernous reservoir.

\subsection{Establishment of mathematical model}

The dimensionless definitions of dimensionless pressure, dimensionless time, channelling coefficient, fracture-cave to matrix storage capacity ratio and fracture-cave storage capacity ratio were made:

$$
\begin{aligned}
& \psi_{j D}=\frac{k_{f} h}{1.842 \times 10^{-3} q B \mu}\left(\psi_{i}-\psi_{j}\right), j=f, m \\
& t_{D}=\frac{3.6 k_{f} t}{\left(\phi_{f} \mu C_{f}+\phi_{m} \mu C_{m}\right) r_{w}^{2}} \\
& \lambda=\alpha \frac{k_{m} r_{w}^{2}}{\mu} ; \omega=\frac{\phi_{f} C_{f}}{\phi_{m} C_{m}+\phi_{f} C_{f}} \\
& \omega_{v}=\frac{\phi_{v} C_{v}}{2\left(\phi_{f} C_{f}+\phi_{v} C_{v}\right)} ; r_{D}=r / r_{w}
\end{aligned}
$$

The dimensionless equations are as follows:

$\frac{1}{r_{D}} \frac{\partial}{\partial r_{D}}\left(r_{D} \frac{\partial \psi_{f D}}{\partial r_{D}}\right)+\lambda\left(\psi_{m D}-\psi_{f D}\right)=\omega \frac{\partial \psi_{f D}}{\partial t_{D}}$

Matrix diffusion equation:

$$
\lambda\left(\psi_{m D}-\psi_{f D}\right)+(1-\omega) \frac{\partial \psi_{m D}}{\partial t_{D}}=0
$$

Initial conditions:

$$
\psi_{j D}\left(r_{D}, t_{D}=0\right)=0, j=f, m
$$

The outer boundary conditions of the banding:

$$
\left.\frac{\partial \psi_{f D}}{\partial r_{D}}\right|_{r_{e D}}=0
$$

Cave area equation:

$$
\begin{aligned}
& \left.\psi_{f D}\right|_{R_{D}}-\psi_{w D}=\left.S R_{D} \frac{\partial \psi_{f D}}{\partial r_{D}}\right|_{R_{D}} \\
& \frac{V_{D}}{24 A_{D}} \omega_{v} \frac{d \psi_{w D}}{d t_{D}}=\left.R_{D} \frac{\partial \psi_{f D}}{\partial r_{D}}\right|_{R_{D}}+1
\end{aligned}
$$

\subsection{Establishment of Laplace mathematical model}

According to the definition of Laplace transform, the dimensionless equation is transformed into a Laplace space expression:

Diffusion equation:

$$
\begin{aligned}
& \frac{1}{r} \frac{\partial}{\partial r_{D}}\left(r_{D} \frac{\partial \bar{\psi}_{f D}}{\partial r_{D}}\right)+\lambda\left(\bar{\psi}_{m D}-\bar{\psi}_{f D}\right)=\omega s \bar{\psi}_{f D} \\
& \lambda\left(\bar{\psi}_{m D}-\bar{\psi}_{f D}\right)+(1-\omega) s \bar{\psi}_{m D}=0
\end{aligned}
$$

Initial conditions:

$\bar{\psi}_{j D}\left(r_{D}, s\right)=0, j=f, m$

Closed outer boundary conditions:

$\left.\frac{\partial \bar{\psi}_{f D}}{\partial r_{D}}\right|_{r_{e D}}=0$

Cave area equation:

$\left.\bar{\psi}_{f D}\right|_{R_{D}}-\bar{\psi}_{w D}=\left.S R_{D} \frac{\partial \bar{\psi}_{f D}}{\partial r_{D}}\right|_{R_{D}}$
$\frac{V_{D}}{24 A_{D}} \omega_{v} u \bar{\psi}_{w D}=\left.R_{D} \frac{\partial \bar{\psi}_{f D}}{\partial r_{D}}\right|_{R_{D}}+\frac{1}{u}$

General solution:

$\bar{\psi}_{w D}=A k_{0}\left(r_{D} \sqrt{u}\right)+B I_{0}\left(r_{D} \sqrt{u}\right)$

External boundary conditions:

$\left.\frac{\partial \bar{\psi}_{w D}}{\partial r}\right|_{r_{D e}}=0$

Substitution:

$-\sqrt{u} A k_{1}\left(r_{D e} \sqrt{u}\right)+B I_{1}\left(r_{D e} \sqrt{u}\right) \sqrt{u}=0$

Solution:

$A=\frac{I_{1}\left(r_{D e} \sqrt{u}\right) B}{k_{1}\left(r_{D e} \sqrt{u}\right)}$

The general solution is as follows:

$\bar{\psi}_{w D}=a B k_{0}\left(r_{D} \sqrt{u}\right)+B I_{0}\left(r_{D} \sqrt{u}\right)$

Substitute into the cave equation:

$$
\begin{aligned}
& a B k_{0}\left(r_{D V} \sqrt{u}\right)+B I_{0}\left(r_{D V} \sqrt{u}\right)-\left(\bar{\psi}_{w D}+\frac{R_{D V} \lambda}{u}\right)= \\
& S R_{D V}\left[-a B \sqrt{u} k_{1}\left(r_{D V} \sqrt{u}\right)+B \sqrt{u} I_{1}\left(r_{D V} \sqrt{u}\right)\right] \\
& \frac{V_{D}}{24 A_{D}} \omega_{v} \frac{d \psi_{w D}}{d t_{D}}=\left.R_{D} \frac{\partial \psi_{f D}}{\partial r_{D}}\right|_{R_{D}}+1
\end{aligned}
$$

Solution:

$B=\frac{\bar{\psi}_{W D}+R_{D V} \lambda / u}{a k_{0}\left(r_{D V} \sqrt{u}\right)+I_{0}\left(r_{D V} \sqrt{u}\right)-S R_{D V} \sqrt{u}\left[I_{1}\left(r_{D V} \sqrt{u}\right)-a k_{1}\left(r_{D V} \sqrt{u}\right)\right]}$

Make:

$b=a k_{0}\left(r_{D V} \sqrt{u}\right)+I_{0}\left(r_{D V} \sqrt{u}\right)-S R_{D V} \sqrt{u}\left[I_{1}\left(r_{D V} \sqrt{u}\right)-a k_{1}\left(r_{D V} \sqrt{u}\right)\right]$

There are: 


$$
B=\frac{\bar{\psi}_{w D}+\frac{R_{D V} \lambda}{u}}{b}
$$

Substitute into the karst cave equation:

$\frac{V_{D}}{24 A_{D}} \omega_{v} u \bar{\psi}_{w D}=$

$R_{D V}\left[-a \sqrt{u} \frac{\bar{\psi}_{w D}+R_{D V} \lambda}{b} k_{1}\left(r_{D V} \sqrt{u}\right)+\frac{\bar{\psi}_{w D}+R_{D V} \lambda}{b} \sqrt{u} I_{1}\left(r_{D V} \sqrt{u}\right)\right]+\frac{1}{u}$

Solution:

$$
\bar{\psi}_{w D}=\frac{b+\lambda R_{D V}^{2} \sqrt{u} I_{1}\left(r_{D V} \sqrt{u}\right)-\lambda a R_{D V}^{2} \sqrt{u} k_{1}\left(r_{D V} \sqrt{u}\right)}{u\left[b \frac{V_{D}}{24 A_{D}} \omega_{v} u+a R_{D V} \sqrt{u} k_{1}\left(r_{D V} \sqrt{u}\right)-R_{D V} \sqrt{u} I_{1}\left(r_{D V} \sqrt{u}\right)\right]}
$$

Cave type capacity response model:

$$
q=\frac{2 \pi k_{f} h}{B \mu \psi_{f D}}\left(p_{i}-p_{f}\right)
$$

\section{Mathematical model of cavernous seepage through drilling}

It can be seen from the seepage pattern diagram of vuggy carbonate reservoir that the seepage pattern of this type of well can be divided into three categories: karst cave radial flow, fracture linear flow and boundary quasi-steady flow.

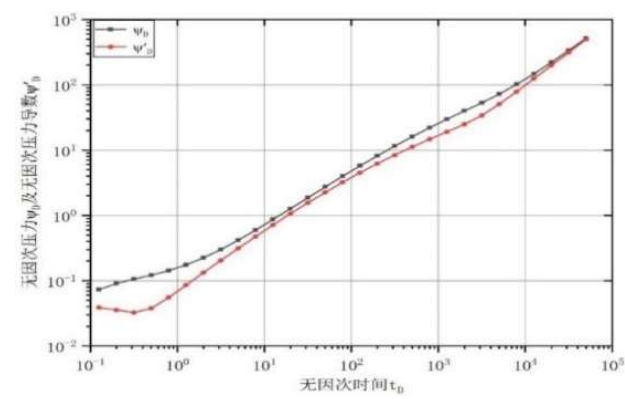

Figure 2. The seepage model of cavern-fracture carbonate reservoir.

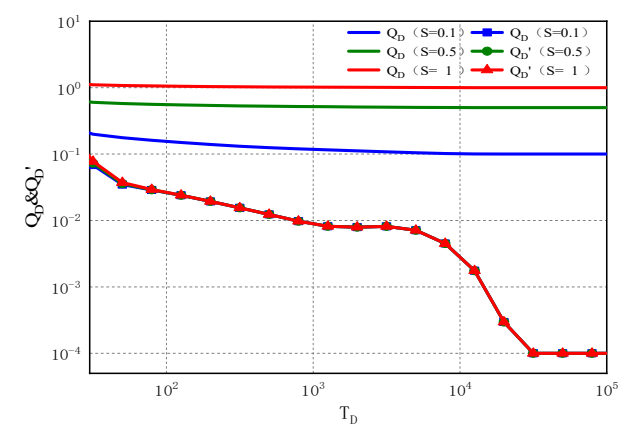

Figure 3. Relation diagram of influence of skin coefficient on productivity of cave-fracture carbonate reservoir (log-log coordinates)

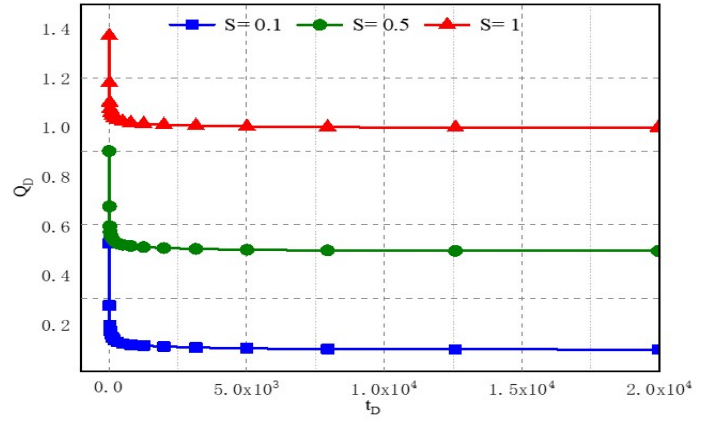

Figure 4. Effect of skin coefficient on productivity of vuggefracture carbonate reservoir (Cartesian coordinates)

Fig. 3 is the skin factor of hole-seam type carbonate reservoir capacity log-log influence diagram, Fig. 4 is the skin factor of hole-seam type carbonate reservoir capacity cartesian coordinate effect diagram, skin factor of holeseam type mainly embodies the influence of carbonate reservoir production at the beginning of the row in phases, the skin factor, the greater the production decline rate is lower, The larger the value of dimensionless yield curve is, the derivative curve of middle and late yield basically coincides.

Fig. 5 are seam wellbore storage coefficient for the hole type carbonate reservoir capacity log-log influence diagram, Fig. 6 seam wellbore storage coefficient for the hole - type carbonate reservoir capacity cartesian coordinate effect diagram, wellbore storage coefficient of seam hole - type of carbonate reservoir production effect is mainly embodied in the stage of high rank of the former, wellbore storage coefficient, the faster the yield decline rate is, the larger the dimensionless yield curve value is, and the middle and late production curves basically coincide with each other without obvious influence.

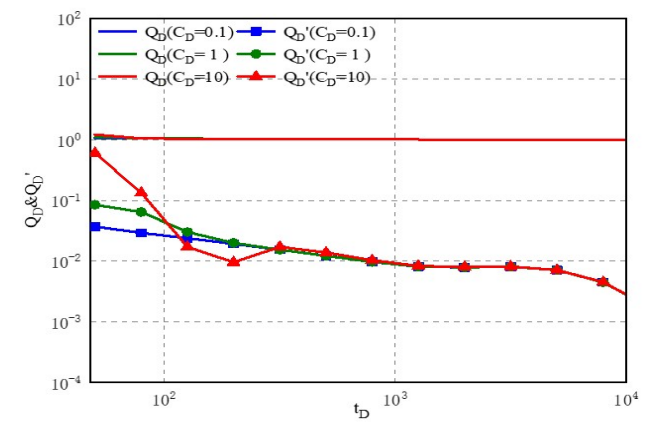

Figure 5. Influence of wellbore reservoir coefficient on productivity of cave-fracture carbonate reservoir (log-log coordinates) 


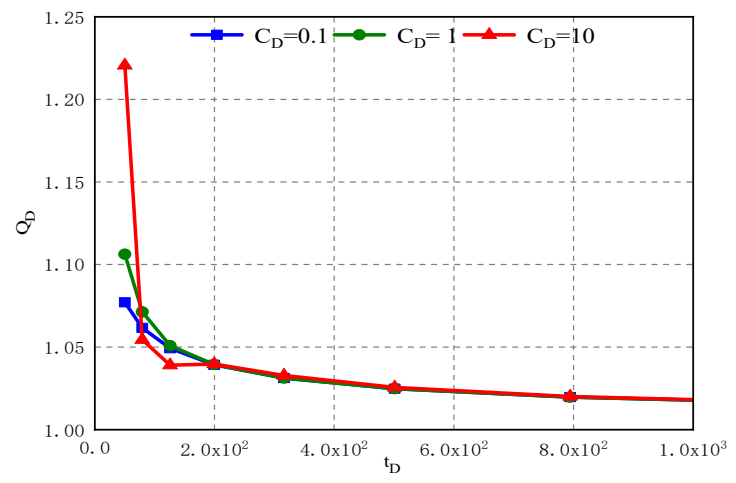

Figure 6. Relation diagram of influence of wellbore reservoir coefficient on productivity of cave-fracture carbonate reservoir (Cartesian coordinates)

\section{Conclusion}

This paper to carry out the buried hill type seam hole carbonate reservoir geological model based on simplified, starting from the basic principle of seepage flow mechanics, establish buried seam hole type carbonate reservoir productivity prediction model and analyze the factors affecting capacity, through Z6 block of single well productivity verification on the spot of this paper to establish the mathematical model of reliability is higher. The results of influence factor analysis show that the larger the storage capacity ratio is, the greater the dimensionless yield curve value is. The higher the interflow coefficient, the greater the dimensionless production curve value. The larger the wellbore reservoir coefficient is, the faster the production decline rate is. The larger the skin factor, the higher the dimensionless yield.

\section{References}

1. Xia Tian, Study on productivity evaluation of buried hill reservoir [D]. Northeast Petroleum University, 2015.

2. Wen Huaizhong, Evaluation of development effect and optimization of production allocation of YM32 carbonate reservoir [D]. Southwest Petroleum University, 2014.

3. Wan Yizhao, Liu Yuewu. Three-dimensional discrete fracture-cavity numerical well test model for fracture-cavity reservoirs [J]. Chinese Journal of Theoretical and Applied Mechanics, 2015,47(06):1000-1008.

4. Liu Jian, Liu Yuetian, Liang Wuquan, et al. Study on out-of-step injection and production of fractured oil reservoirs in carbonate buried hill $[\mathrm{J}]$. Science, Technology and Engineering, 2013(36):49-54.

5. LIN Xing, Study on numerical simulation method of buried-hill fractured-vuggy carbonate reservoir [D]. Southwest Petroleum University, 2014.

6. Feng Jinde, Study on seepage theory and reservoir engineering application of fractured low permeability reservoir [D]. China University of Petroleum (Beijing), 2007.
7. $\mathrm{Xu}$ Tonghao, Geng Shaoyang, Li Yao, $\mathrm{Hu}$ Gang.Productivity evaluation model of buried-hill fractured-vug carbonate reservoir and analysis of influencing factors $[\mathrm{J}]$. Petrochemical Industry Application, 2020,39(09):40-46

8. Kang Bo, Simulation and application of multi-scale reservoir flow in deep karst condensate gas reservoir [D]. Southwest Petroleum University, 2020. 